\title{
Study of the Forces Acting on the Animal in the Installation for Fixing with Veterinary Treatments
}

\section{B A Doronin, O | Detistova, D || Gritsay, | | Shvetsov, and E V Kulaev}

Stavropol State Agrarian University, Russia

\section{Abstract}

The study of the forces acting on the animal in the installation for fixing with veterinary treatments. The most time consuming processes in service animals are zootechnical and veterinary treatment of sheep. During the year, it is necessary to carry out more than ten such treatments of each animal, which requires a lot of labor. Almost all animal treatments such as feed to the operator's workplace and their fixation in a convenient position for him requires significant physical effort of the operator. Therefore, today the technologies and technical means should be created to reduce labor costs for various treatments. The relevance of the problem is due to the lack of theoretical

Corresponding Author:

B A Doronin

Received: 25 October 2019

Accepted: 15 November 2019

Published: 25 November 2019

Publishing services provided by Knowledge E

(c) B A Doronin et al. This article is distributed under the terms of the Creative Commons

Attribution License, which permits unrestricted use and redistribution provided that the original author and source are credited.

Selection and Peer-review under the responsibility of the AgroSMART 2019 Conference Committee. foundations and experimental data for the creation of technological equipment for fixing sheep in zootechnical and veterinary treatments. The purpose of the study is the theoretical and experimental justification of the installation for fixing sheep in zootechnical and veterinary treatments with the justification of the existing efforts on the animal, excluding injury. Developed installation and presented a scheme with two of the conveyor belts forming the grooved shape When designing the installation, the main focus was on the justification of structural elements and modes of operation from the viewpoint of eliminating the possibility of injury to the animal. The experimental studies have confirmed the correctness of the obtained analytical dependences. The obtained results will enable designers to create equipment for the fixation of sheep at the zootechnical and veterinary treatments, precluding injury to the animal and reducing labor costs.

Keywords: sheep breeding, zootechnical processing, veterinary processing, installation for fixing sheep, belt conveyors.

\section{Introduction}

Sheep breeding is a complex production and economic system aimed at meeting the needs of the population in foodstuffs and industry in agricultural raw materials. In the Stavropol region, sheep breeding traditionally was a key industry in the agricultural sector of the economy. However, over the past decade in the region and in the country, the production of wool and lamb has decreased significantly, and the production of sheep breeding products has become unprofitable [1, 2]. 
The most labour-intensive processes in the animal servicing are zootechnical and veterinary treatments of sheep (shearing, branding, valuation, hoof trimming, vaccination, etc.) being an integral part of the production engineering in sheep breeding. During the year, it is necessary to carry out more than ten such treatments of each sheep which requires a lot of labour. For example, labour inputs to carry out zoo-andveterinary treatments for a year are 1360...1440 man-hour on one thousand females $[3,4]$. The industry does not manufacture rigging for zoo-and-veterinary treatments, with the exception of tanks for chemical solutions $[5,6]$.

Practically during all zoo-and veterinary treatments, an animal delivery to an operator and their fixation in a convenient position require considerable physical effort of the operator, which often causes injury to animals. Rough labour and its social unattractiveness is a characteristic feature of a shepherd's profession at the present time. Therefore, today technologies and means of production for sheep breeding should be created being alternative to traditional technologies with a glance to the safety of animals on the basis of high-performance, robotic equipment. Alongside with the solution of the main goal -- profitable production of sheep breeding products -- these projects have to solve social problems. In this direction, the work is being carried out both in our country and abroad [7, 8].

The experimental studies of the efforts acting on an animal in the installation for fixation and sheep delivery during zoo-and-veterinary treatments were carried out at research laboratory of the FGBOU VO "Stavropol State Agrarian University".

\section{Materials and Methods}

To improve the quality of zootechnical and veterinary treatments of sheep (shearing, branding, valuation, hoof trimming, vaccination, etc.) and to increase labour productivity, we have developed and tested an installation for fixation and delivery of animals (Fig. 1). When developing the installation, the main attention was paid to the justification of structural elements and modes of operation in terms of eliminating the possibility of injury to the animal [1].

The operation of the installation is as follows. An animal enters the receiving part of the installation from the "entrance" and is delivered to the operator's area by conveyors to perform zoo-and-veterinary treatments. The location of belt conveyors at an angle to the horizon provides lifting the supporting limbs of the animal from the ground, and the $V$-shaped location of conveyors with an angle $\varphi$ fixes the sheep with weigh $m_{1}$ under the action of gravity $\left(m_{1} g\right)$. Taking into account the main dimensions of a sheep (female: 
length up to $940 \mathrm{~mm}$, ram -- up to $1300 \mathrm{~mm}$ ), the length of the installation is accepted within $3.0 \mathrm{~m}$, at the same time two sheep can be on the installation: one at the entrance, the second -- at the exit.

The main source of effort acting in the system under consideration is the gravity of the animal being moved.

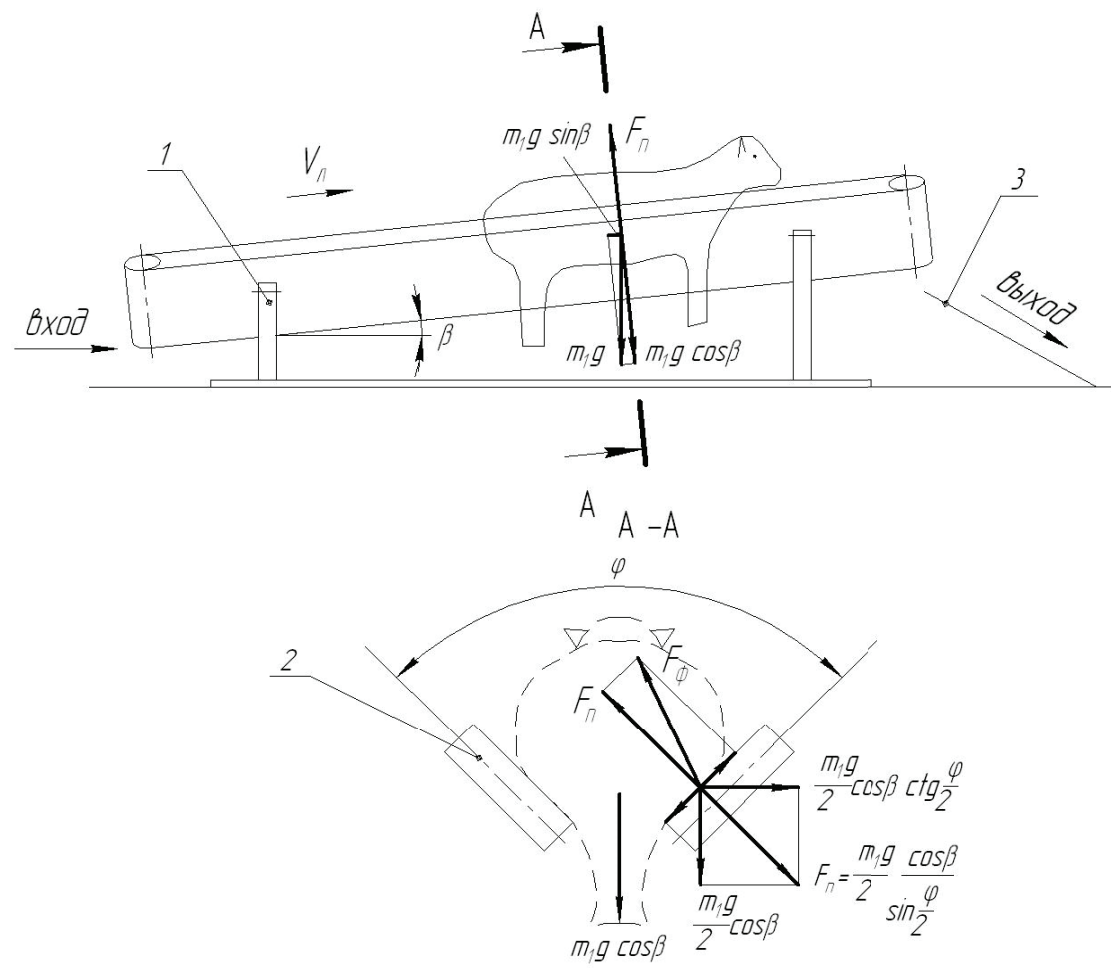

Figure 1: Diagram of the installation for fixation and delivery of sheep during zoo-and-veterinary treatments and the forces acting on the animal, by way of example of one conveyor: 1 -- installation frame; 2 -- belt conveyors; 3 -- inclined board for sheep leaving.

\section{Results and Discussion}

To study the resistance forces of belts motion of conveyors, the gravity of the linear mass of sheep is determined (Fig. 2).
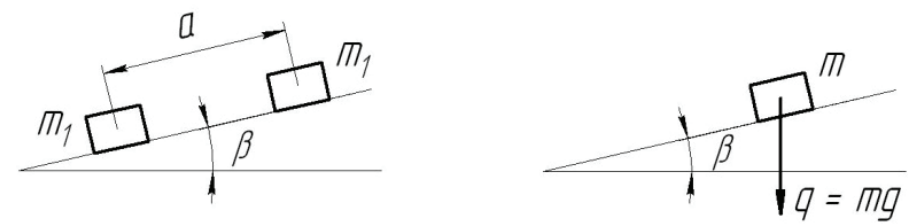

Figure 2: Diagram for the calculation of the linear gravity.

$$
m=m_{1} / a q=m g
$$


where $m_{1}$-- weight of sheep, kg;

$a$-- distance between sheep, $\mathrm{m}$;

$m$-- linear weight of sheep, $\mathrm{kg} / \mathrm{m}$;

$g$-- acceleration of free fall, $\mathrm{m} / \mathrm{s}^{2}$;

$q$-- gravity of the linear weight, $N / m$.

To determine the reinforcements arising during the movement of sheep by two conveyors [2], two planes are considered: the plane of gravity I-I and the plane II-II perpendicular to the axis of the conveyors (Fig. 3).

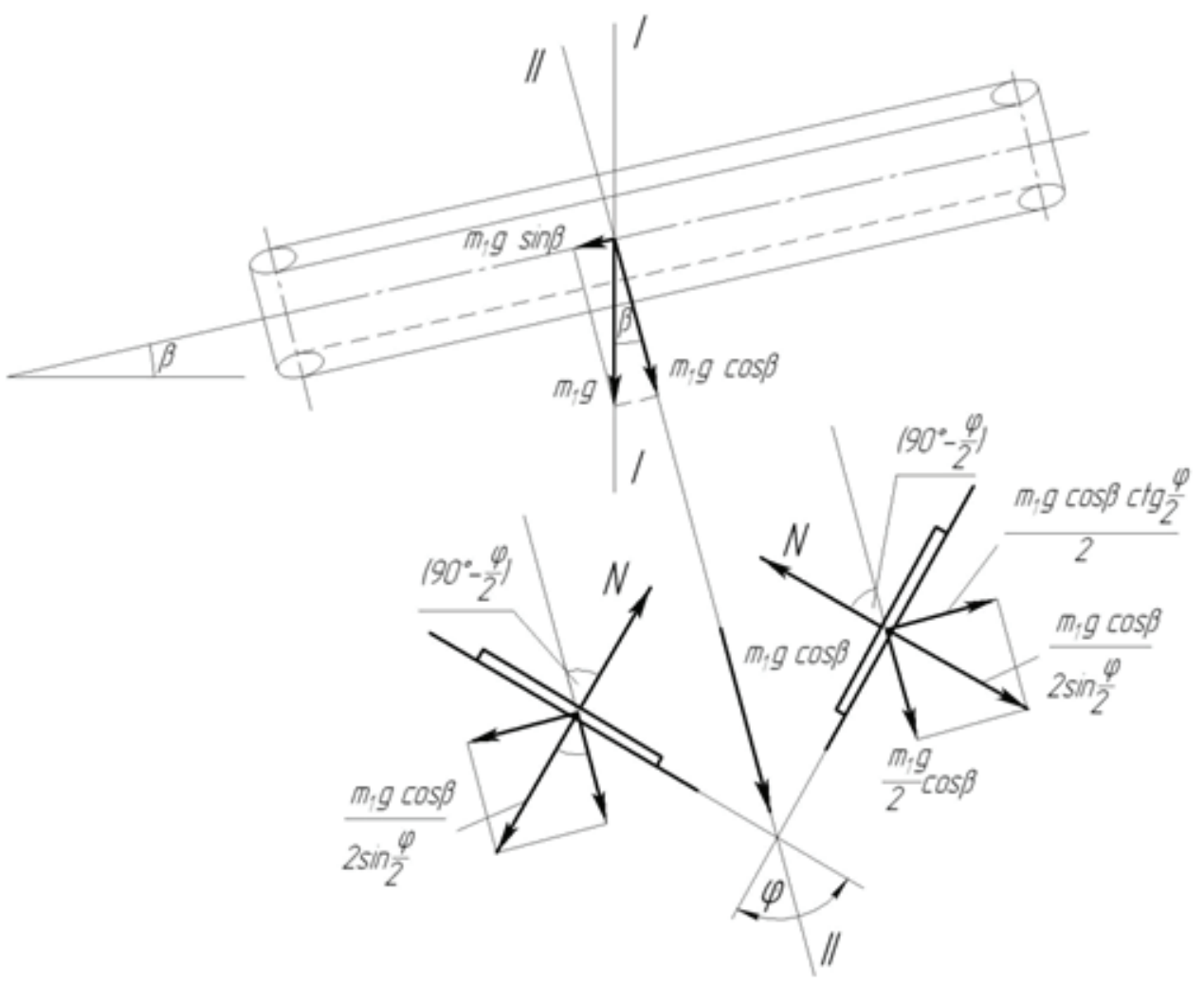

Figure 3: Diagram of the forces in the installation when fixing and moving sheep.

To determine the linear forces of the normal pressure $N$ on the belts of the conveyors, the acting forces are projected onto the plane II-II [1].

$$
2 N \cdot \cos \left(90^{\circ}-\frac{\varphi}{2}\right)-q \cos \beta=0, \quad \text { from where } N=\frac{q \cos \beta}{2 \sin \frac{\varphi}{2}}=\frac{m_{1} g \cos \beta}{2 a \sin \frac{\varphi}{2}}
$$

The component reinforcement of the normal pressure $N$ for one conveyor is determined in the plane II-II (Fig. 3) through the weight of a transported sheep

$$
\frac{q}{2} \cos \beta=\frac{m_{1} g \cdot \cos \beta}{2 a} \text { and } \frac{m_{1} g \cdot \cos \beta \cdot \operatorname{ctg} \frac{\varphi}{2}}{2 a}
$$

The total resistance reinforcement of the loaded branch of the conveyor will be

$$
T=T_{\text {suppl }}+T_{\text {mov }}+T_{\text {idl.run }}+T_{j}
$$


where $\mathbf{T}$-- the total tractive effort of belt, $\mathbf{N} ; \mathbf{T}_{\text {suppl }}$--- tractive effort of the belt to rise sheep, $\mathrm{N}, T_{\text {sup } p l}=\frac{q}{2} \sin \beta \cdot L=\frac{m_{1} g}{2 a} \sin \beta \cdot L$, here $L$-- length of the conveyor, $\mathrm{m} ; \mathbf{T}_{\text {mov }}$ -- tractive effort of sheep movement, $\mathrm{H}, T_{\text {mov }}=\frac{q \cos \beta}{2 \sin \frac{\varphi}{2}} \cdot L \cdot \omega=\frac{m_{1} g \cos \beta}{2 a \sin \frac{\varphi}{2}} \cdot L \cdot \omega$, here $\omega$

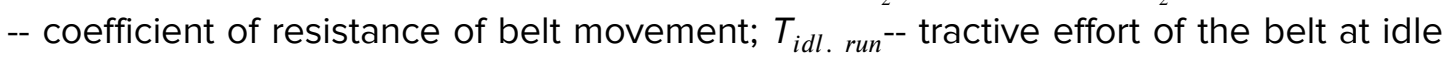
running, $\mathrm{N}$.

The tractive effort at idle running will be determined by the force of the belt movement

$$
T_{i d l . r u n}=2 m_{0} g \cdot \cos \beta \cdot \sin \frac{\varphi}{2} \cdot L \cdot \omega,
$$

where $m_{o}$-- the linear mass of the conveyor belt, $\mathrm{kg} / \mathrm{m}$.

Tractive effort to overcome the inertial forces $T_{j}$ can be determined by the introduction of the concepts of conditional performance of the conveyor and conditional second work.

The conditional performance of the conveyor $Q(\mathrm{t} / \mathrm{h})$ is $Q=3.6 \mathrm{~m} V_{l}=3.6 \frac{m_{1}}{a} V_{l}$, where $V$-- belt speed, $\mathrm{m} / \mathrm{s}$.

The second mass $m_{c}(\mathrm{~kg} / \mathrm{s})$ is determined $m_{c}=\frac{Q}{3.6}$.

Then the second work of the inertial forces is equal to $A_{c}=\frac{Q\left(V_{l}^{2}-V_{0}^{2}\right)}{2 \cdot 3.6}=T_{j} V_{l}$, whence $T_{j}=\frac{Q\left(V_{l}^{2}-V_{0}^{2}\right)}{2 \cdot 3.6 V_{l}}$, where $V_{o}-$ the initial speed of the sheep, $\mathrm{m} / \mathrm{s}$.

Substituting instead of $Q=3.6 \frac{m_{1}}{a} V_{l}$, we get $T_{j}=\frac{m_{1}\left(V_{l}^{2}-V_{0}^{2}\right)}{2 a}$.

The expressions for $T, T, T_{x x}$ and $\mathbf{T}_{j}$ are substituted in (4) and the formula of the total resistance reinforcement of the loaded branch of the conveyor will be of the form

$$
T=\frac{m_{1} g}{2 a} \sin \beta \cdot L+\frac{m_{1} g \cdot \cos \beta}{2 a \cdot \sin \frac{\varphi}{2}} \cdot L \cdot \omega+2 m_{0} g \cdot \cos \beta \cdot \sin \frac{\varphi}{2} \cdot L \cdot \omega+\frac{m_{1}\left(V_{\pi}^{2}-V_{0}^{2}\right)}{2 a}
$$

Analyzing the expression (5) for the parameters $m_{1} \approx 100 \mathrm{~kg} ; L \approx 3 \ldots 4 \mathrm{M} ; \beta \approx 20^{\circ} . .30^{\circ}$; $\varphi=90^{\circ} ; \omega=0.3 ; m_{0} \approx 2 \ldots 3 \mathrm{kgr} ; V_{l} \approx 1 \mathrm{~m} / \mathrm{s} ; a \approx 1 \ldots 2 \mathrm{M}$, it is seen that $\frac{T_{\text {idl. } r u n}+T_{j}}{T_{\text {sup } p l}+T_{\text {mov }}} \approx 0.08 \ldots 0.1$, therefore, the dependence (5) can be represented as follows

$$
T=\frac{K_{u} m_{1} g L}{2 a} \cdot\left(\sin \beta+\frac{\cos \beta}{\sin \frac{\varphi}{2}} \cdot \omega\right)
$$

where $\mathbf{K}_{u}$-- the coefficient taking into account the resistance reinforcement of the idle running and inertial forces, $K_{u} \approx 1.1-1.2$

The experimental studies were carried out on a specially designed and manufactured installation; the diagram and general view are shown in Figure 4.

The installation (Fig. 4) consists of longitudinal units 1 on which racks 2 are installed. At the top of the racks 2 , supporting surfaces are floated -- left 3 and right 4 . The rubberfabric conveyor belts 5 are attached along the length of last ones. The free sides of the supporting surfaces are supported through a semicircle 6 , a ball 7 , and a disk 8 on a 
strain- gauge transducer 9 . The latter is floated on a telescopic rod 10 connected to the longitudinal bar 11 which has the ability to be fixed on the racks 2 at different height. Left 3 and right 4 supporting surfaces are set at an angle $\varphi$ with the help of the telescopic rod 10. In this case, the angle between the supporting surfaces 3, 4 and rods 10 should be $90^{\circ}$ by changing the length of the rod 10 and reinstalling vertically on the racks 2 of the bar 11. The distance between the lower free sides of the supporting surfaces 3 and 4 is regulated by two telescopic rods 12 connected to the racks 2 . Fixation of the distance is carried out by the screw 13 . The model 14 of the animal or the animal itself is placed between the supporting surfaces 3 and 4 . The model 14 of the animal is made in size and weight shaped like the animal body of sustained density. The model is covered with sheepskin to ensure the actual friction coefficient between the model of the animal and the conveyor belt $[2,8]$.

The angle $\beta$ is set by lifting the ends of the longitudinal beams 1 in the vertical plane.

Measurement of the force acting on the animal was carried out with the help of a measuring unit.
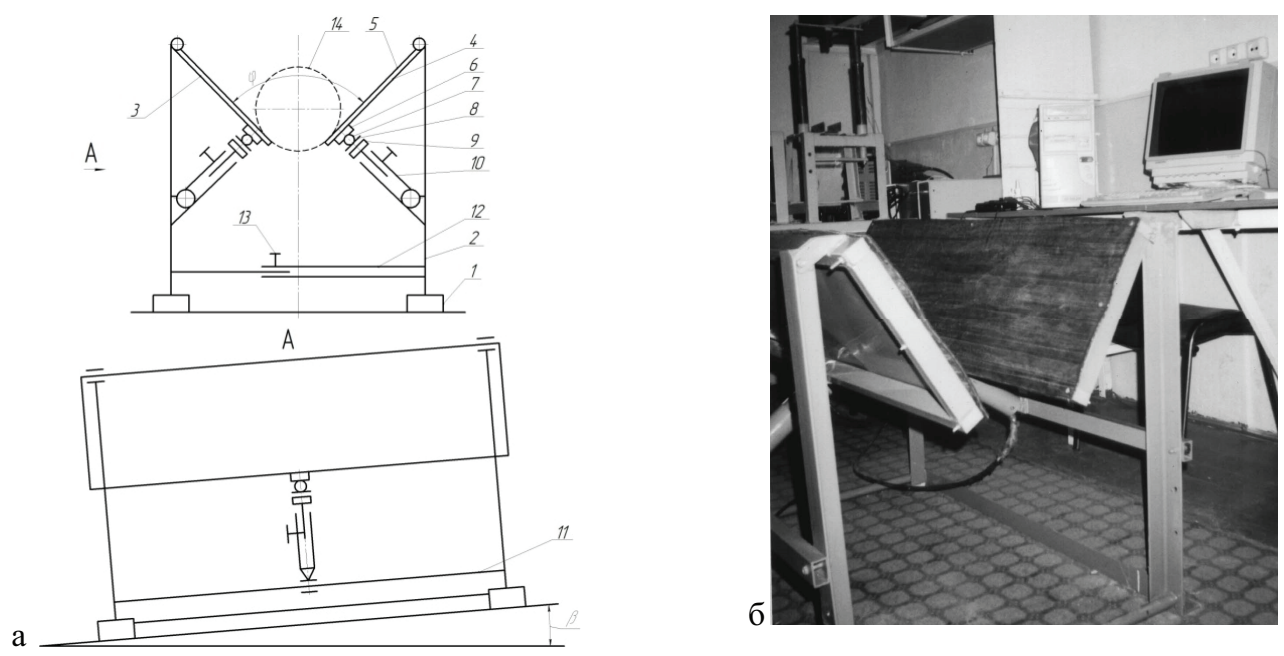

Figure 4: Diagram $(A)$ and general view (b) of the installation to determine the forces, acting on an animal in the installation for sheep fixation and delivery during zoo- and-veterinary treatments.

Graphic dependencies (Fig. 5) made on the basis of the experimental data show that the resistance force of the belt increases with decreasing angle between the conveyors and increasing the coefficient of belt movement on the flooring.

A significant increase of the resistance force occurs when the angle between the conveyors decreases, especially less than 90 degrees.

Therefore, in the installation design in order to reduce expenditure of energy for the drive, it is advisable to have conveyors at an angle of 90 degrees and more, and the supporting surfaces of the conveyor belts are performed using roller carriages [2]. 


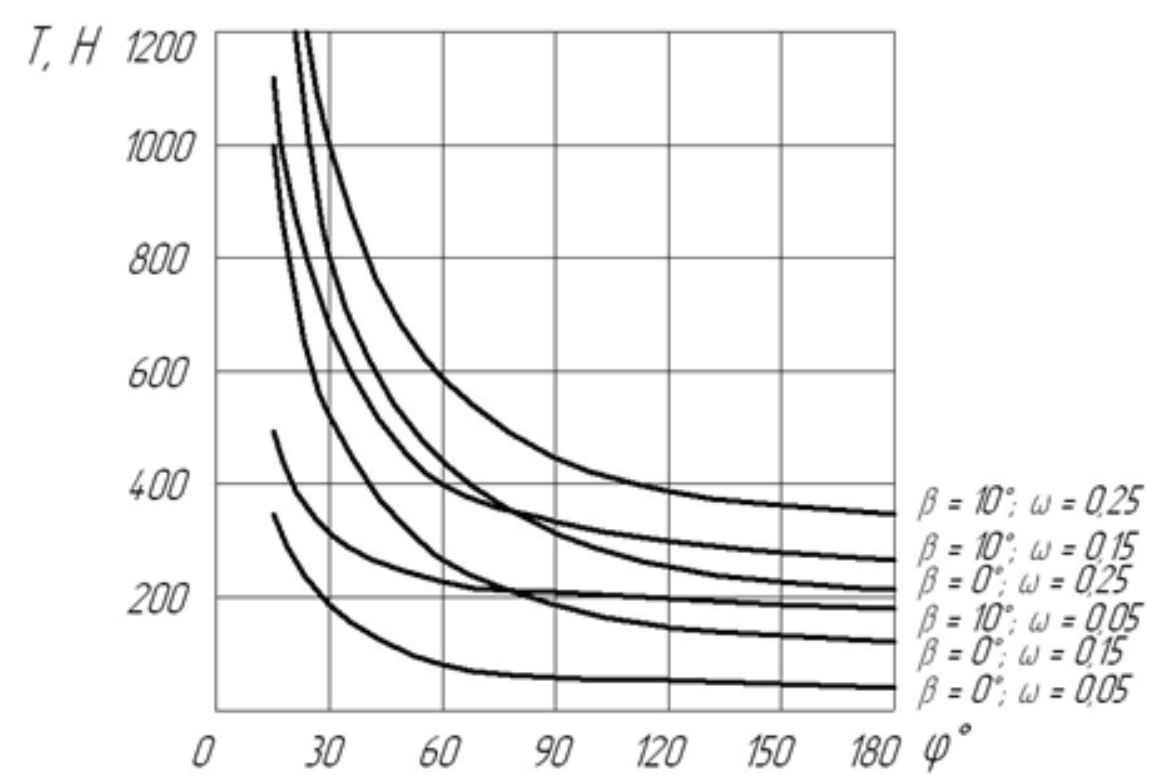

Figure 5: Resistance force of the conveyor belt depending on the installation characteristics.

The drive power of the conveyors of the installation for zoo-and-veterinarian treatments of sheep is determined on the basis of the obtained dependence

$$
P=\frac{K_{u} m_{1} g L}{2 a} \cdot\left(\sin \beta+\frac{\cos \beta}{\sin \frac{\varphi}{2}} \cdot \omega\right) \cdot V_{\pi}
$$

where $\mathbf{V}$-- the speed movement of the belt, $V_{l}=0.3 \ldots 0.6 \mathrm{~m} / \mathrm{s}$.

Fig. 6 shows the diagram of change of the required drive power of conveyors depending on the parameters of the installation and the animal weight.

In this case, the concurrence of the values of power $\mathrm{P}$ at the point "A" indicates that when you change one argument or another (installation parameter or sheep weight), the other arguments are accepted as constant with the maximum possible values.

Taking into account the presence of two sheep at the same time (entry and exit), the required drive power is about 0.8...1.0 kW. When increased angle $\varphi$ between the conveyors, required drive power of the stations is reduced, fixation properties of $V$ shaped positioned conveyor are also decreased. Therefore, the most optimal angle is recommended to accept $\varphi=90^{\circ}$. The other parameters influence power in direct proportion to, and it is recommended to use over the range: $m=90 . .120 \mathrm{~kg} ; \beta=6^{\circ} . .10^{\circ}$; $\mathbf{v}_{l}=0.3 \ldots .0 .6 \mathrm{~m} / \mathrm{s}$.

\section{Conclusion}

The experimental studies of the changes in the power of the conveyor drive depending on the structural and kinematic parameters of the installation show that the relative 


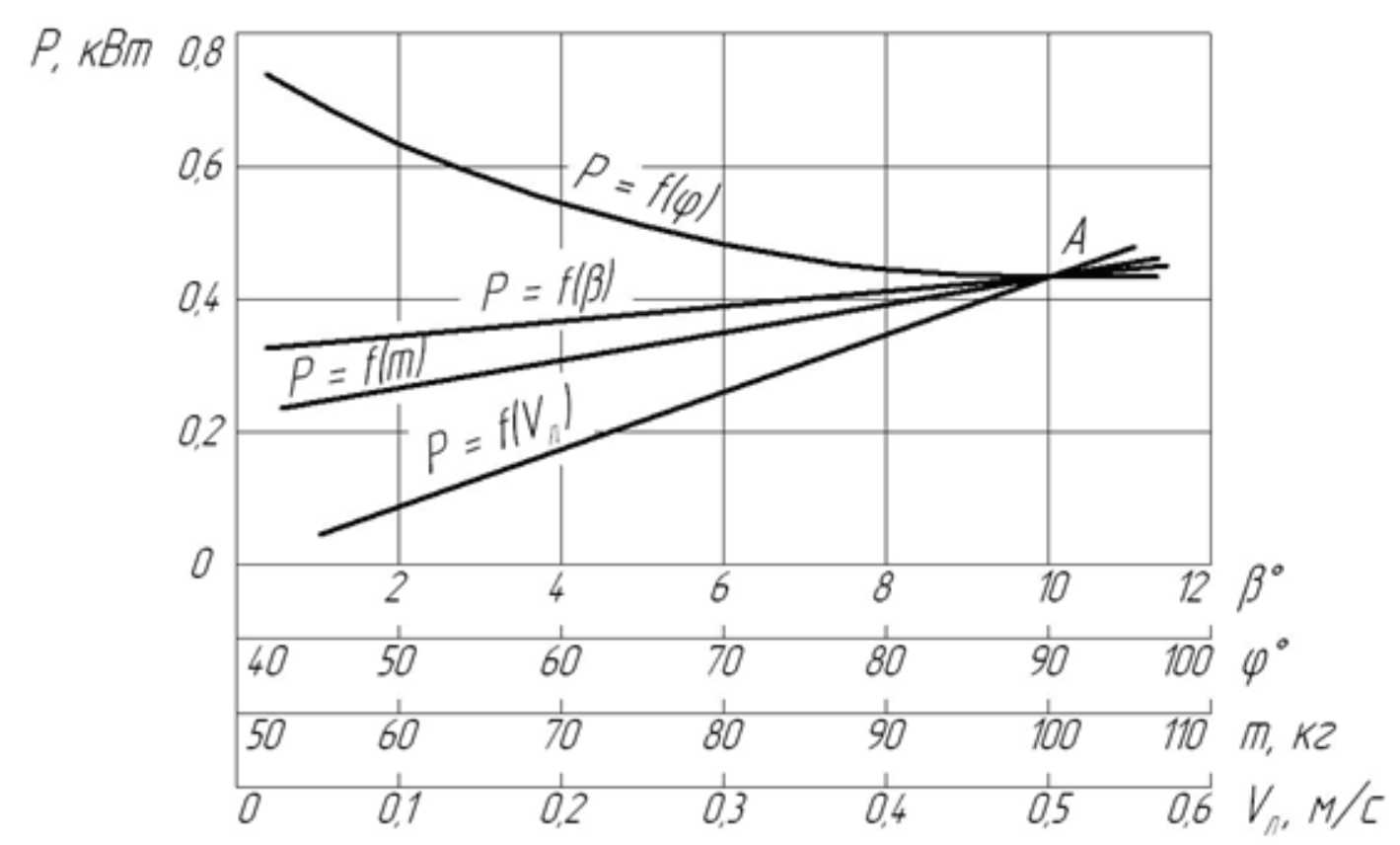

Figure 6: The drive power of conveyors depending on the installation parameters and animal.

deviations of the values calculated theoretically by the proposed analytical dependences and obtained experimentally are not more than $14 \%$. It validates the analytical expressions obtained in the theoretical study and their adequate description of the real process. The obtained results are intended for engineers of agricultural machines to design process equipment for sheep breeding. The obtained results will allow creating equipment for fixation and delivering of sheep during zoo-and-veterinary treatments excluding injury of animals.

\section{References}

[1] Trukhachev, V.I., Doronin, B.A., Detistova, O.I., Gritsay, D.I. (2016). Designing apparatus for fixing sheep at veterinary treatment. Research Journal of Pharmaceutical, Biological and Chemical Sciences, vol. 7, no. 6, pp. 2309--2314.

[2] Doronin, B.A., Lebedev, A.T., Detistova, O.I., Gritsay, D.I. (2016). Upgrading of squeeze chutes for Sheep. Research Journal of Pharmaceutical, Biological and Chemical Sciences, vol. 7, no. 3, pp. 1895--1901.

[3] Chistyakov, N.D. (2005). Low-cost technology in sheep breeding. Russian cattle breeding, no 1, pp. 24--25.

[4] Moroz, V.A., Trukhachev, V.I. (2006). Perspective technologies for the production of sheep products in the southern region of Russia. Collection of scientific papers of the GNU VNIIMZH, vol. 16, Part 1, pp. 117--125. Podolsk. 
[5] Mirzoyants, Yu.A. (2006). Directions of mechanization and automation of sheep breeding. Engineering in agriculture, no. 1, pp. 12--14.

[6] Mirzoyants, Yu.A. (2002). New technical means for mechanization of technological processes in sheep breeding. Technological and technical support of crop production and livestock production: a collection of scientific papers VIM, vol. 4. Moscow: VIM, pp. 207--216.

[7] Alekseenko, NP. (2006). Ways to increase the reliability and prospects for the production of shearing equipment in Russia. Collection of scientific papers of the GNU VNIIMZH, vol. 16, part 2. Podolsk, pp. 119--122.

[8] Suyunchaliev, R.S. (2005). Results and directions of research on the mechanization of sheep breeding and the creation of a new technique. Scientific and technical progress in animal husbandry -- promising resource-saving technologies: a collection of scientific papers, part 2, vol. 15. Podolsk: GNU VNIIMZH, pp. 206--211. 